\title{
Vitamin E or coenzyme Q10 administration is not fully advantageous for heart mitochondrial function in diabetic goto kakizaki rats
}

\author{
Paulo J. Oliveira ${ }^{\mathrm{a}, *}$, Raquel Seica ${ }^{\mathrm{b}}$, Dario L. Santos ${ }^{\mathrm{c}}$, Anabela P. Rolo ${ }^{\mathrm{a}}$, \\ Vilma A. Sardao ${ }^{a}$, Fernanda M.L. Ferreira ${ }^{a}$, Carlos M. Palmeira ${ }^{a}$, \\ Maria S. Santos ${ }^{\mathrm{a}}$, Antonio J.M. Moreno ${ }^{\mathrm{a}}$ \\ ${ }^{a}$ Center for Neurosciences and Cellular Biology of Coimbra, Department of Zoology, University of Coimbra, P-3004-517 Coimbra, Portugal \\ ${ }^{\mathrm{b}}$ School of Medicine, Center for Neurosciences and Cellular Biology of Coimbra, University of Coimbra, Coimbra, Portugal \\ ${ }^{\mathrm{c}}$ Department of Environmental and Biological Engineering, University of Tras-os-Montes, Vila Real, Portugal
}

Received 19 November 2003; received in revised form 23 January 2004; accepted 5 February 2004

\begin{abstract}
The heart is one of the organs affected during the later stages of diabetes. Mitochondrial function has already been proposed to be affected during the course of diabetes. Nevertheless, little information is known concerning the impact of antioxidants in heart mitochondria of a milder model for diabetes, such as the Goto-Kakizaki (GK) rat, where mitochondrial function appears ameliorated. The objective of this work was to test if injections of Vitamin E and Coenzyme Q10, alone and in combination, were able to modify mitochondrial performance in the hearts of GK rats.

Several aspects of mitochondrial function were measured, such as the respiratory control ratio and the electric potential, as well as the mitochondrial accumulation of Vitamin E and Coenzymes Q9 and Q10. We observed that only Vitamin E appeared to have a positive impact on the mitochondrial phosphorylation efficiency and on mitochondrial performance, namely on the ability to generate the electric transmembrane potential in the presence of supra-physiological calcium concentrations. Vitamin E administration also increased the mitochondrial concentration of Coenzyme Q10. None of the treatments was able to reverse the diabetic phenotype in GK rats. We conclude that in this model of mild hyperglycemia, administration of antioxidants may have a marginal positive impact on mitochondrial function.
\end{abstract}

(C) 2004 Elsevier B.V. and Mitochondria Research Society. All rights reserved.

Keywords: $\Delta \Psi$, Mitochondrial electric potential; VitE, Vitamin E; CoQ, Coenzyme Q; GK rat, Goto-Kakizaki rat; TPP ${ }^{+}$, Tetraphenylphosphonium cation; MPT, Mitochondrial permeability transition; MPTP, Mitochondrial permeability transition pore; ADP/O, nmol ADP consumed per natm $\mathrm{O}$ consumed

\footnotetext{
* Corresponding author. Tel.: +351-239-480210; fax: +351239-480217.

E-mail address: pauloliv@ci.uc.pt (P.J. Oliveira).
}

\section{Introduction}

Diabetes mellitus is one of the most common metabolic diseases. The complications associated with this condition are often responsible for 
a decreased quality of life in many patients. Mortality in the diabetic population is primarily due to cardiovascular disease, which is not dissimilar from the non-diabetic population. However, the diabetic population has a greater probability to suffer from cardiovascular problems and heart failure than the general population (Pierce et al., 1997; Chaudhuri and Hopkins, 2000). The cardiovascular mortality rate is more than doubled in men and more than quadrupled in women with diabetes when compared with their non-diabetic counterparts. Mechanisms leading to vascular perturbations such as atherosclerosis (King and Wakasaki, 1999) have been proposed to explain the complications associated with this pathology (Chaudhuri and Hopkins, 2000). Endothelial dysfunction associated with diabetes may lead to impaired vasodilatation, causing vascular and cardiac dysfunction (De Vriese, 2000).

Mitochondria, being cellular powerhouses, can play a dual role in diabetic pathology. Mutations in the mitochondrial DNA are associated with the development of diabetes (Gerbitz et al., 1996; Perucca-Lostanlen et al., 2000) and diabetes was itself shown to modify mitochondrial function (Kristal et al., 1997). Functional mitochondria in pancreatic $\beta$-cells are needed to provide the necessary ATP to sustain insulin release into blood (Gerbitz et al., 1995; Anderson, 1999), thus illustrating the importance of mitochondrial bioenergetics in the process of diabetes.

Type 2 (non-insulin dependent diabetes mellitusNIDDM) is one of the most common metabolic diseases in man. The development of model animals carrying this pathology is also of crucial importance to study the biochemical changes observed at the organism level. The Goto-Kakizaki (GK) rat has been designed as a NIDDM model (Goto et al., 1975, 1988) and used in several metabolic studies related to this pathology.

Surprisingly, previous studies with GK rats showed an improvement of hepatic mitochondrial bioenergetics (Ferreira et al., 1999a,b) and mitochondrial antioxidant capacity, including increased levels of vitamin E (Ferreira et al., 1999c), suggesting specific changes in mitochondria. It appears that those modifications acted as an adaptation to the hyperglycemic condition. Previous work has also described an enhanced calcium loading capacity in heart mitochondria from GK rats (Oliveira et al., 2001).
Diabetes and associated complications are often associated with increased oxidative stress (Baynes, 1991; Baynes and Thorpe, 2000; Schmidt and Stern, 2000). Extensive literature has debated the use of antioxidants to counteract some complications associated with the hyperglycemic condition. Coenzyme Q (CoQ) or ubiquinone (2,3,-dimethoxy5-methyl-6-multiprenyl-1,2-benzoquinone) is a redox-active and lipophilic substance present in most cellular membranes. It consists of a quinone head attached to a chain of isoprene units numbering 9 or 10 (CoQ9 or CoQ10) in the different mammal species. CoQ plays several roles in cells. Probably, the most well known function of CoQ in cells is electron transfer in the mitochondrial electron transport chain. Coenzyme $Q$ is able to transfer electrons from Complex I or II to Complex III (Ernster and Dallner, 1995). Furthermore, its reduced form was proposed as an antioxidant (Ernster and Dallner, 1995). It was previously described that coenzyme Q10 administration was able to decrease protein oxidation and increase the antioxidant potential in the rat (Lamson and Plaza, 2002). Some reports also confirmed some clinical advantage of coenzyme $\mathrm{Q}$ against diabetes-derived complications (for a review see Lamson and Plaza, 2002).

Vitamin E ( $\alpha$-tocopherol, VitE) is the major lipid soluble chain-breaking antioxidant found in the plasma and tissues (Burton and Traber, 1990). A joint action of both coenzyme $Q$ and vitamin $E$ against cellular oxidative damage has also been proposed (Kagan et al., 1990).

By using an animal model for a mild form of diabetes, the GK rat, we investigated whether CoQ10, VitE or both compounds together could indeed ameliorate cardiac mitochondrial function. So far, no report in the literature is available concerning the possible benefits of such treatments in a mitochondrial population that appears to have enhanced functions in this model for mild hyperglycemia.

\section{Materials and methods}

\subsection{Materials}

All reagents and chemicals used were of the highest grade of purity commercially available. 
CoQ10 and VitE were dissolved in soybean oil (Sigma S7381) prior to in vivo injections.

\subsection{Animals}

Male GK rats (4 weeks) were maintained in our local colony (Laboratory Animal Research Center, University Hospitals, Coimbra) with ad libitum access to food (AO4-Panlab, Spain)) and water at $\mathrm{pH} 4.5$. Room temperature was kept at $22-24{ }^{\circ} \mathrm{C}$ and the moisture at $50-60 \%$. The animals were subjected to a $12 \mathrm{~h}$ day/night cycle. The research procedure was carried out in accordance with the European Requirement for Vertebrate Animal Research.

\subsection{Animal treatment}

GK rats were randomly divided into four groups: Soybean Oil alone (2 ml/Kg i.p., control), Vitamin E (200 mg/Kg, i.p., VitE), Coenzyme Q10 (20 mg/Kg, i.p., CoQ10) and Coenzyme Q10 + Vitamin E ( $200 \mathrm{mg} / \mathrm{Kg}$, i.p.VitE plus $20 \mathrm{mg} / \mathrm{Kg}$, i.p. CoQ10 CoQ10 + VitE). There were no statistically significant differences concerning initial body weights between the four experimental groups.

\subsection{Biochemical analyses}

Animals were injected three times a week during seven weeks. Initial evaluations were performed before the start of the treatment. Final evaluations were conducted $24 \mathrm{~h}$ after the last injection. Blood glucose concentration (occasional glucose) was determined from the tail vein using a commercial glucometer (Glucometer-Elite, Bayer). The glycated hemoglobin $\left(\mathrm{HbA}_{1 \mathrm{c}}\right)$ was determined at the time of sacrifice through ionic exchange chromatographic assay (Abbott IMx Glicohemoglobin, Abbott Laboratories, Portugal).

\subsection{Isolation of mitochondria from rat heart}

Twenty four hours after last injection, animals were sacrificed and their heart excised. Rat heart mitochondria were prepared as previously described (Oliveira et al., 2000). Mitochondrial protein content was determined by the biuret method calibrated with BSA.

\subsection{Measurement of mitochondrial transmembrane potential}

The mitochondrial transmembrane potential $(\Delta \Psi)$ was estimated with a tetraphenylphosphonium cation $\left(\mathrm{TPP}^{+}\right)$electrode according to the equation of Kamo et al. (1979), without correction for the 'passive' binding contribution of $\mathrm{TPP}^{+}$to the mitochondrial membranes (as the purpose of the experiment was to show relative changes in the potential rather than absolute values). A matrix volume of $1.1 \mu \mathrm{l} / \mathrm{mg}$ protein was assumed and valinomycin was used to calibrate the basal line. Reactions were carried out at $25^{\circ} \mathrm{C}$ in $1 \mathrm{ml}$ of the reaction media $(200 \mathrm{mM}$ sucrose,

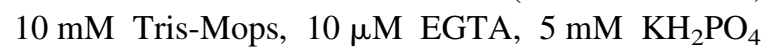
and $2 \mu \mathrm{M}$ rotenone, $\mathrm{pH}$ 7.4) supplemented with $1 \mu \mathrm{M}$ $\mathrm{TPP}^{+}$and $0.5 \mathrm{mg}$ of mitochondria. Mitochondria were energized with $8 \mathrm{mM}$ succinate. ADP-induced depolarization was calculated as the maximum difference observed in membrane potential measured after ADP addition. Phosphorylation lag phase was measured as the time in seconds the mitochondrial suspension took to phosphorylate the added ADP (that is, the time between mitochondrial depolarization and repolarization).

\subsection{TPP $^{+}$uptake in the presence of calcium}

Mitochondrial $\mathrm{TPP}^{+}$uptake was performed using a $\mathrm{TPP}^{+}$-selective electrode, using a $\mathrm{AgCl}$ reference electrode. Mitochondrial protein $(0.5 \mathrm{mg})$ was suspended in $2 \mathrm{ml}$ of reaction buffer $(200 \mathrm{mM}$ sucrose, $10 \mathrm{mM}$ TRIS-MOPS, $10 \mu \mathrm{M}$ EGTA and $5 \mathrm{mM}$ $\mathrm{KH}_{2} \mathrm{PO}_{4}, \mathrm{pH} 7.4,25^{\circ} \mathrm{C}$ ), supplemented with $2 \mu \mathrm{M}$ rotenone and $0.5 \mu \mathrm{M} \mathrm{TPP}^{+}$. Sequential additions of $0.5 \mu \mathrm{M} \mathrm{TPP}{ }^{+}$were made in order to calibrate the system. Succinate was added to the mitochondrial suspension after a total of $3 \mu \mathrm{M} \mathrm{TPP}^{+}$in the buffer, in the presence of $100 \mu \mathrm{M}$ calcium. In each assay, the calibration was used to calculate the $\mathrm{TPP}^{+}$accumulated inside mitochondria in the presence of calcium. Succinate was the substrate used as this allowed a fully reduced pool of pyridine nucleotides.

\subsection{Mitochondrial oxygen consumption}

Oxygen consumption of isolated heart mitochondria was monitored polarographically with a Clark oxygen 
electrode connected to a suitable recorder simultaneously with the $\Delta \Psi$ measurement. Reactions were carried out, at $25^{\circ} \mathrm{C}$, in $1 \mathrm{ml}$ of the reaction media composed by $200 \mathrm{mM}$ sucrose, $10 \mathrm{mM}$ Tris-Mops, $10 \mu \mathrm{M}$ EGTA, $5 \mathrm{mM} \mathrm{KH}_{2} \mathrm{PO}_{4}$ and $2 \mu \mathrm{M}$ rotenone, $\mathrm{pH}$ 7.4. Mitochondria were suspended at a concentration of $0.5 \mathrm{mg} / \mathrm{ml}$ in the respiratory medium and energized with $8 \mathrm{mM}$ succinate. This substrate was used in order to avoid possible artifacts related to loss of activity of complex I during mitochondrial isolation. ADP (100 nmol) was added to induce state 3 respiration. The Respiratory Control Ratio (RCR) was calculated as the ratio between state 3 and state 4 respiration. The ADP/O was calculated as the number of nmol ADP phosphorylated by natom oxygen consumed.

\subsection{Extraction and quantification of $\mathrm{CoQ} 9, \mathrm{CoQ} 10$, and vitamin $E$}

Aliquots of mitochondria containing $1 \mathrm{mg}$ of protein $/ \mathrm{ml}$ were extracted according to the method described by Takada et al. (1984). The extract was evaporated to dryness under a stream of $\mathrm{N}_{2}$ and resuspended in absolute ethanol. CoQ content was determined by reverse-phase HPLC (Spherisorb RP18, S5ODS2 column). Samples were eluted with methanol:heptane $(10: 2 \mathrm{vol} / \mathrm{vol})$ at a flow rate of $2 \mathrm{ml} / \mathrm{min}$. Detection was performed by an ultraviolet (UV) detector at $269 \mathrm{~nm}$. Vitamin E was extracted and quantified by following the method described by Takayanagi et al. (1980). The extract was evaporated to dryness under a stream of $\mathrm{N}_{2}$ and resuspended in $n$-hexane. Vitamin $\mathrm{E}$ content was determined by reverse-phase HPLC $(4.6 \times 200 \mathrm{~mm}$; Spherisorb S10w column). Samples were eluted with $n$-hexane supplemented with $0.9 \%$ methanol at a flow rate of $1.5 \mathrm{ml} / \mathrm{min}$. Detection was performed by a UV detector at $287 \mathrm{~nm}$.

\subsection{Statistical analysis}

The results are presented as the mean \pm SEM of 5-8 independent experiments. Statistical analysis was performed by using One Way Analysis of Variance (ANOVA) and the Dunnet pos-test. A $P$ value $<0.05$ was considered statistically significant.

\section{Results}

Table 1 presents the data regarding animal weight determination and biochemical analyses of occasional glycemia and glycated hemoglobin. As seen, none of the treatment groups was significantly different from the control group (vehicle-injected rats). At the time of sacrifice, normal (non-diabetic) Wistar rats of the same age showed occasional glycemia values of $84 \pm 2 \mathrm{mg} / \mathrm{dl}$ and glycated hemoglobin values of $5.26 \pm 0.09 \%$, thus showing that GK rats used in this study suffered from a mild form of hyperglycemia (occasional glycemia values of $147 \pm 13 \mathrm{mg} / \mathrm{dl}$ and glycated hemoglobin values of $5.93 \pm 0.07 \%$ ). Both values were different from non-diabetic Wistar rats $(P<0.001, n=5)$.

After isolation of the cardiac mitochondrial fraction, we assessed mitochondrial respiratory parameters. The values obtained for the RCR were a good indication of mitochondrial integrity (Table 2). No differences between groups were observable in the RCR value, although we detected significant decreases in state 4 respiration in VitE, CoQ and $\mathrm{CoQ}+$ VitE-treated groups when compared with the vehicle (Table 2). No differences were found

Table 1

Animal weight determination and biochemical analyses in occasional glycaemia and glycated haemoglobin

\begin{tabular}{lcccc}
\hline & Oil (control) & Vitamin E & Coenzyme Q10 & Coenzyme Q10 + Vitamin E \\
\hline Final body weight $(\mathrm{g})$ & $235.30 \pm 6.35$ & $242.5 \pm 4.25$ & $232.60 \pm 9.0$ & $237.70 \pm 7.41$ \\
Weight gain $(\mathrm{g})$ & $178.0 \pm 6.47$ & $182.60 \pm 5.70$ & $171.96 \pm 5.80$ & $175.10 \pm 4.03$ \\
Occasional glycaemia (mg/dl) & $147 \pm 13$ & $164 \pm 16$ & $156 \pm 10$ & $145 \pm 7$ \\
HbA1c $(\%)$ & $5.93 \pm 0.07$ & $5.8 \pm 0.14$ & $5.65 \pm 0.09$ & $5.64 \pm 0.11$ \\
\hline
\end{tabular}

The results are presented as mean \pm SEM of 5-8 independent experiments. Occasional glycaemia was measured at the time of animal sacrifice. HbA1c, glycated haemoglobin. 
Table 2

Mitochondrial function parameters and biochemical analyses from the four experimental groups

\begin{tabular}{lcccc}
\hline & Oil (control) & Vitamin E & Coenzyme Q10 & Coenzyme Q10+ Vitamin E \\
\hline State 4 (natms O/min/mg prot) & $110.4 \pm 5.8$ & $73.7 \pm 5.0 \#$ & $82.3 \pm 4.7^{*}$ & $89.1 \pm 10.0^{*}$ \\
State 3 (natms O/min/mg prot) & $296.0 \pm 19.9$ & $224.7 \pm 31.9$ & $236.8 \pm 26.0$ & $247.0 \pm 33.2$ \\
RCR & $2.7 \pm 0.1$ & $2.6 \pm 0.1$ & $2.6 \pm 0.1$ & $2.6 \pm 0.1$ \\
ADP/O & $1.4 \pm 0.1$ & $1.9 \pm 0.2^{*}$ & $1.6 \pm 0.1$ & $1.6 \pm 0.2$ \\
$\Delta \Psi(\mathrm{mV})$ & $230.4 \pm 1.2$ & $233.3 \pm 1.0$ & $232.1 \pm 1.7$ & $225.5 \pm 4.8$ \\
ADP-induced depolarization (mV) & $41.0 \pm 2.20$ & $35.6 \pm 2.6$ & $39.2 \pm 1.12$ & $42.71 \pm 5.17$ \\
Coenzyme Q10 (pmol/mg) & $124.10 \pm 11.04$ & $187.2 \pm 14.77^{*}$ & $148.48 \pm 8.78$ & $178.40 \pm 20.33$ \\
Vitamin E (nmol/mg) & $1.38 \pm 0.10$ & $1.30 \pm 0.06$ & $1.11 \pm 0.08$ & $1.32 \pm 0.10$ \\
\hline
\end{tabular}

The results are presented as mean \pm SEM of 5-8 independent experiments. ADP $(100 \mathrm{nmol})$ was added to induce state 3 respiration. The Respiratory Control Ratio (RCR) was calculated as the ratio between state 3 and state 4 respiration. The ADP/O was calculated as the number of nmol ADP phosphorylated by natom oxygen consumed. *, $P<0.05$ vs. oil; \#, $P<0.01$ vs. oil.

regarding state 3 . The significant increase in the ADP/O value in the VitE-treated animals (Table 2), is worthy of attention and will be confirmed later with a different technique. We determined the levels of CoQ10 and VitE in the cardiac mitochondrial fraction in order to determine the enrichment of those fractions (Table 2). Surprisingly, only a mitochondrial enrichment on CoQ10 was found in the VitE group. No other differences were found. When measuring Coenzyme Q9 (data not shown), no differences were again found.

Mitochondrial generation of transmembrane electric potential $(\Delta \Psi)$ was also determined in the four experimental groups, as well as the ADP-induced depolarization. No differences were again observable between treatment groups (Table 2).

Measurements of the phosphorylation lag phase provided valuable information. This parameter represents the time required for the mitochondrial suspension to phosphorylate the amount of ADP added to the reaction buffer. As is apparent (Fig. 1), heart mitochondria from VitE-treated animals showed a shorter lag-phase, which signifies a faster phosphorylation activity. Nevertheless, VitE, when coadministrated with CoQ10, did not have the same effect.

One particular aspect of mitochondrial dysfunction is a reduction in calcium loading capacity. We performed experiments in which the objective was to determine the amount of $\mathrm{TPP}^{+}$that heart mitochondria from the four groups were capable of loading in a buffer containing calcium. The mitochondrial permeability transition (MPT) is a condition that acts as a redox sensor of the inner mitochondrial environment. As described later, increased mitochondrial oxidative stress can induce the MPT in the presence of calcium. Our experiments were a way of indirectly assessing mitochondrial ability to load calcium by measuring the maximal mitochondrial retention of $\mathrm{TPP}^{+}$. An early occurrence of the calcium-dependent MPT would be related to a lower $\mathrm{TPP}^{+}$accumulation (since it would lead to membrane depolarization and inability to accumulate the TPP cation). Rats treated with VitE showed a higher ability to generate membrane potential when incubated with calcium (100 $\mu$ M, Fig. 2). In contrast, cardiac

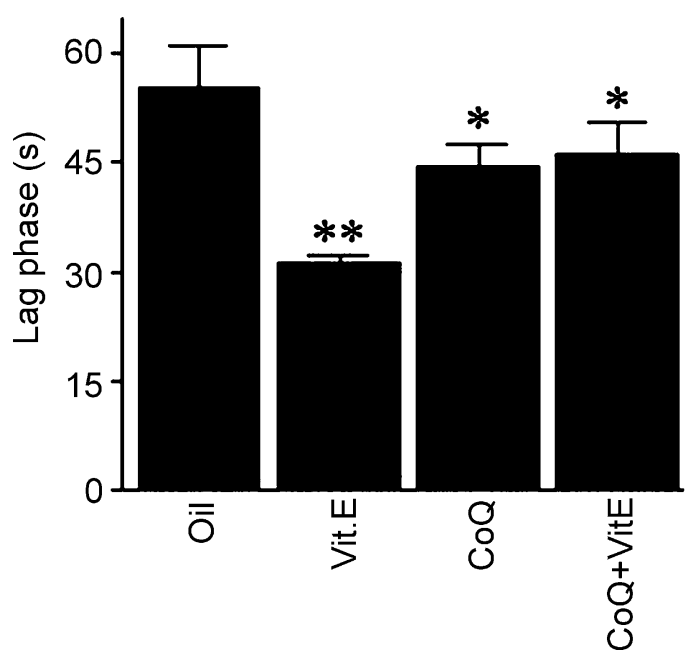

Fig. 1. Phosphorylative lag phase. Lag phase was measured as described in Section 2. The results are presented as mean \pm SEM of $5-8$ independent experiments. $* P<0.05$ vs. VitE, $* * P<0.001$ vs. control (oil-injected animals). 


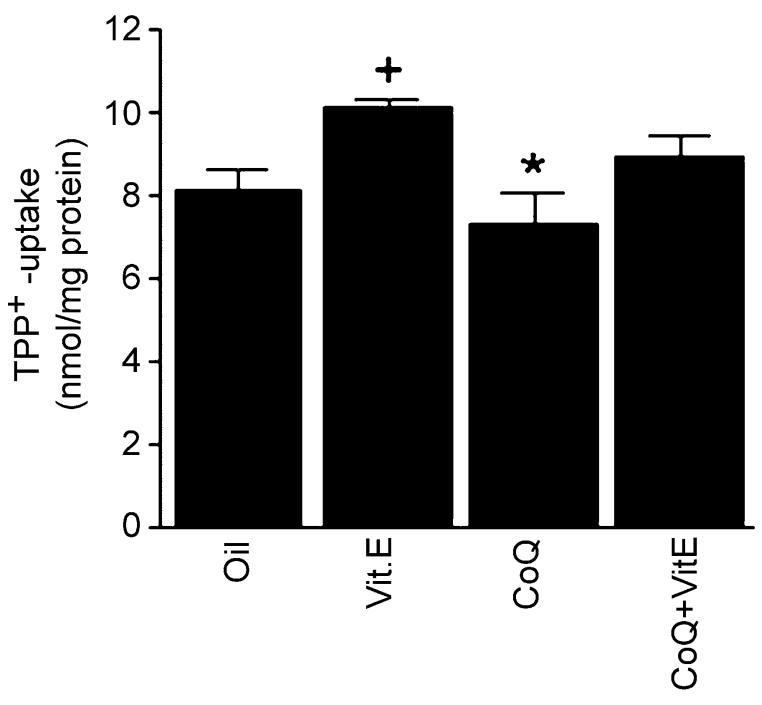

Fig. 2. $\mathrm{TPP}^{+}$accumulation in the presence of $100 \mu \mathrm{M}$ calcium, measured by using a $\mathrm{TPP}^{+}$-selective electrode. Sequential additions of $0.5 \mu \mathrm{M} \mathrm{TPP}{ }^{+}$were made in order to calibrate the system. Succinate was added to the mitochondrial suspension after a total of $3 \mu \mathrm{M} \mathrm{TPP}^{+}$in the buffer. In each assay, the calibration was used to calculate the $\mathrm{TPP}^{+}$accumulated inside mitochondria in the presence of calcium. Succinate was the substrate used as this allowed a fully reduced pool of pyridine nucleotides. The results are presented as mean \pm SEM of $5-8$ independent experiments. $* P<0.05$ vs. VitE, $+P<0.001$ vs. control (oil-injected animals).

mitochondria isolated from rats injected simultaneously with VitE and CoQ10 did not differ from controls regarding this parameter.

\section{Discussion}

Despite many studies on the impact of antioxidants on diabetic complications (for a review see Lamson and Plaza, 2002), no studies are available concerning the effects of such compounds on cardiac mitochondrial performance in diabetic GK rats. To study this subject, we used GK rats, an animal model for hereditary diabetes and moderate hyperglycemia (Goto et al., 1975, 1988). We injected CoQ10 and VitE, alone and in combination, and determined changes in cardiac mitochondrial function.

From our results, VitE appeared more promising regarding the improvement of mitochondrial function, despite the fact that no changes whatsoever were observed in the diabetic phenotype. Three aspects of mitochondrial function were ameliorated in VitE injected animals. Two of them were complementary and were related to the mitochondrial phosphorylative system. It appears that heart mitochondria from VitEinjected animals were able to phosphorylate ADP in a faster way (as revealed by the shorter lag phase), although no changes in the RCR value were obtained, and by consuming a lower amount of oxygen in the process (revealed by the ADP/O ratio, a measurement of the mitochondrial phosphorylation efficiency). The other aspect concerned an increased ability to generate membrane potential in the presence of supra-physiological calcium concentrations. It is noticeable that the effect of VitE was absent when animals were injected with both compounds, which was a rather unexpected result.

In a first analysis, the most surprising result was the absence of mitochondrial accumulation of CoQ10 in CoQ10-injected animals (Table 2). Nevertheless, it is not the first time that such a result has been observed. Ibrahim et al. (2000) observed that CoQ10 oral administration did not alter the levels of that compound in the heart. Nevertheless, the authors observed a dose-dependent sparing effect of VitE in CoQ10 levels in several organs, but not in the heart. From Table 2, one can see that the administration of VitE did not cause an increase of VitE in the cardiac tissue but caused instead an increase of CoQ10. Coenzyme Q9, in accordance with previous works (Ibrahim et al., 2000) was also not affected (data not shown). It has previously been demonstrated that oxidative stress inside mitochondria is inversely proportional to the membrane concentration of CoQ10 (Lass et al., 1999). From our results, we can predict that the protective effects of VitE administration as observed were mediated by a sparing effect on CoQ10, as described by other authors (Kagan et al., 1990). Some literature reports that VitE enhances the biosynthesis of coenzyme Q (Donchenko et al., 1991), which can, in fact, explain the increased amounts of CoQ10 found in the mitochondrial membranes of VitE injected animals. It was also previously proposed (Fontaine and Bernardi, 1999) that CoQ10 is inhibitory for MPTP opening, which may help to explain the increment in mitochondrial calcium loading capacity. As far the more efficient ADP phosphorylation seen in VitE-injected animal 
is concerned, the mechanism is still unclear, although some previous reports in the literature confirm that in vitro CoQ supplementation may indeed increase the efficiency of oxidative phosphorylation in isolated heart mitochondria (Battino et al., 1990). Although we did not explore this matter in more detail, it appears that the increased phosphorylative efficiency in the presence of VitE is not directly related to the presence of a hyperglycemic condition. In fact, the effect appears to be due to other mechanisms than mere membrane interactions of VitE since VitE, CoQ and CoQ + VitE all caused a decrease in state 4 respiration which can be indicative of decrease in inner membrane permeability to protons. From those three groups only VitE was able to increase the ADP/O value. A logical question is why VitE and CoQ10 did not show synergistic effects when administrated together. In fact, we observed that the simultaneous administration of both compounds had no effect whatsoever on the several mitochondrial parameters measured. Although rather unexpected, a previous report in the literature suggested that $\mathrm{CoQ}$ and VitE could compete regarding intestinal absorption when administrated together (Kaikkonen et al., 2002).

Although the heart is an organ known to be affected during diabetes-associated complications (Pierce et al., 1997; Chaudhuri and Hopkins, 2000), it was suggested that cardiac dysfunction is dependent upon the degree of diabetes (Feuvray and Lopaschuk, 1997; Paulson, 1997). In fact, we demonstrated that GK rats have a higher calcium loading capacity by means of lower calcium gating of the MPT (Oliveira et al., 2001), which could work as an advantage to those animals. The MPT is linked to the formation and opening of protein pores (the MPT pores) in the inner mitochondrial membrane (Zoratti and Szabò, 1995). MPT pore opening can be caused by excessive mitochondrial calcium accumulation and oxidative stress generation (Kowaltowski et al., 2001), and can lead ultimately to cell death (Kroemer and Reed, 2000). We propose that the recurrent hyperglycemia and oxidative stress in the GK rats (Ihara et al., 1999) could trigger adaptation mechanisms in order to counteract that condition (Oliveira et al., 2001), which would lead to increased mitochondrial function (as described above). Thus, it is predictable that an extra supplementation of some antioxidants will not have such a marked effect since mitochondrial function in this aspect is already ameliorated. This is in contrast to what we would expect to see in other models of more severe diabetes, in which oxidative defences and mitochondrial function may be compromised. Furthermore, as discussed, the heart appears not to accumulate exogenously added VitE and CoQ10 (Ibrahim et al., 2000), despite the idea that Vitamin E supplementation may help increase endogenous CoQ10 levels in that tissue (Ibrahim et al., 2000).

We propose that for milder forms of diabetes in animal models, where apparent mitochondrial adaptations appear to have occurred, the administration of antioxidants will have a very limited positive impact on mitochondrial function, not only due to small cardiac tissue enrichment but also to the already ameliorated mitochondrial function. Nevertheless, VitE showed a more encouraging result. Studies remain to be done in animal models for severe diabetes, such as the streptozotocin-injected animal, where dysfunctional mitochondrial function is observed (Kristal et al., 1997). Despite VitE positive impact, it did not reverse the diabetic phenotype found in GK rats.

In conclusion, in all forms of administration, VitE showed a modest positive impact, possibly through a sparing effect on CoQ10 or by increasing its biosynthesis, as previously reported (Donchenko et al., 1991). This study also demonstrated the administration of CoQ10 and VitE to GK rats does not result in a clear direct enrichment of the compounds in the cardiac mitochondrial fraction and appears not advantageous in the management of diabetes complications.

\section{Acknowledgements}

We thank Prof. João Patrício and co-workers (Maria de Lourdes and Carlos Silva, from the Animal Research Center Laboratory, University Hospitals, Coimbra) for all the help in maintaining the animals. We also thank Dr Teresa Proença (Laboratory of Neurochemistry, University Hospitals, Coimbra) for the measurements of Coenzyme Q10, Q9 and Vitamin E. Paulo J. Oliveira is supported by a Pos-Doc grant 
from the Portuguese Foundation for Science and Technology (SFRH/BPD/8359/2002).

\section{References}

Anderson, C.M., 1999. Mitochondrial dysfunction in diabetes mellitus. Drug Dev. Res. 46, 67-79.

Battino, M., Fato, R., Parenti-Castelli, G., Lenaz, G., 1990. Coenzyme $\mathrm{Q}$ can control the efficiency of oxidative phosphorylation. Int. J. Tissue React. 12(3), 137-144.

Baynes, J.W., 1991. Role of oxidative stress in development of complications in diabetes. Diabetes 40, 405-412.

Baynes, J.W., Thorpe, S.R., 2000. Oxidative Stress in Diabetes. In: Antioxidants in Diabetes Management, Marcel Dekker Inc, New York, pp. 77-91.

Burton, G.W., Traber, M.G., 1990. Vitamin E: antioxidant activity, biokinetics, and bioavailability. Annu. Rev. Nutr. 10, 357-382.

Chaudhuri, D., Hopkins, W.E., 2000. Cardiac Disease in Diabetes Mellitus, Medical Management of Diabetes Mellitus, Marcel Dekker, New York.

De Vriese, A.S., 2000. Endothelial dysfunction in diabetes. Br. J. Pharmacol. 130, 963-974.

Donchenko, G.V., Kruglikova, A.A., Shavchko, L.P., Polishchuk, N.L., 1991. The role of vitamin $\mathrm{e}$ in the biosynthesis of ubiquinone (q) and ubichromenol (qc) in rat liver. Biokhimiia 56(2), 354-360.

Ernster, L., Dallner, G., 1995. Biochemical, physiological and medical aspects of ubiquinone function. Biochem. Biophys. Acta 1271, 195-204.

Ferreira, F.M.L., Palmeira, C.M., Matos, M.J., Seiça, R., Santos, M.S., 1999c. Decreased susceptibility to lipid peroxidation of goto-kakizaki rats: relationship to mitochondrial antioxidant capacity. Life Sci. 65, 1013-1025.

Ferreira, F.M.L., Palmeira, C.M., Seiça, R., Santos, M.S., 1999a. Alterations of liver mitochondrial bioenergetics in diabetic Goto-Kakizaki rats. Metabolism 48, 1115-1119.

Ferreira, F.M.L., Seiça, R., Santos, M.S., Palmeira, C.M., 1999b. Age-related alterations in liver mitochondrial bioenergetics of diabetic Goto-Kakizaki rats. Acta Diabetol. 36, 173-177.

Feuvray, D., Lopaschuk, G.D., 1997. Controversies on the sensitivity of the diabetic heart to ischemic injury: the sensitivity of the diabetic heart to ischemic injury is decreased. Cardiovasc. Res. 34, 113-120.

Fontaine, E., Bernardi, P., 1999. Progress on the mitochondrial permeability transition pore: regulation by complex $\mathrm{I}$ and ubiquinone analogs. J. Bioenerg. Biomemb. 31, 335-345.

Gerbitz, K-D., Gempel, K., Brdiczka, D., 1996. Mitochondria and diabetes-genetic, biochemical, and clinical implications of the cellular energy circuit. Diabetes 45, 113-126.

Gerbitz, K-D., Van den Ouweland, J.M.W., Maassen, J.A., Jaksch, M., 1995. Mitochondrial diabetes mellitus: a review. Biochem. Biophys. Acta 1271, 253-260.

Goto, Y., Kakizaki, M., Masaki, N., 1975. Production of spontaneous diabetic rats by repetition of selective breeding. Tohoku J. Exp. Med. 119, 85-90.
Goto, Y., Suzuki, K., Sasaki, M., Ono, T., Abe, S., 1988. GK rat as a model of non-obese, non-insulin-dependent diabetes. Selective breeding over 35 generations. In: Shafrir, A.R., (Ed.), Frontiers in Diabetes Research: Lessons from Animal Diabetes II, John Libbey, London, pp. 301-303.

Ibrahim, W.H., Bhagavan, H.N., Chopra, R.K., Chow, C.K., 2000. Dietary coenzyme q10 and vitamin e alter the status of these compounds in rat tissues and mitochondria. J. Nutr. 130, 2343-2348.

Ihara, Y., Toyokuni, S., Uchida, K., Odaka, H., Tanaka, T., Ikeda, H., et al., 1999. Hyperglycemia causes oxidative stress in pancreatic beta-cells of GK rats, a model of type 2 diabetes. Diabetes 48(4), 927-932.

Kagan, V., Serbinova, E., Packer, L., 1990. Antioxidant effects of ubiquinones in microsomes and mitochondria are mediated by tocopherol recycling. Biochem. Biophys. Res. Commun. 169 , $851-857$.

Kaikkonen, J., Tuomainen, T.P., Nyyssonen, K., Salonen, J.T., 2002. Coenzyme Q10: absorption, antioxidative properties, determinants, and plasma levels. Free Radic. Res. 36(4), 389-397.

Kamo, N., Muratsugu, M., Hongoh, R., Kobatake, Y., 1979. Membrane potential of mitochondria measured with an electrode sensitive to tetraphenyl phosphonium and relationship between proton electrochemical potential and phosphorylation potential in steady state. J. Membr. Biol. 49, $105-121$.

King, G.L., Wakasaki, H., 1999. Theoretical mechanisms by which hyperglycemia and insulin resistance could cause cardiovascular diseases in diabetes. Diab. Care 22, C31-C37.

Kowaltowski, A.J., Castilho, R.F., Vercesi, A.E., 2001. Mitochondrial permeability transition and oxidative stress. Fed. Eur. Biochem. Sci. Lett. 495, 12-15.

Kristal, B.S., Jackson, C.T., Chung, H-Y., Matsuda, M., Nguyen, H.D., Yu, B.P., 1997. Defects at center P underlie diabetesassociated mitochondrial dysfunction. Free Rad. Biol. Med. 22, $823-833$.

Kroemer, G., Reed, J.C., 2000. Mitochondrial control of cell death. Nat. Med. 6, 513-519.

Lamson, D.W., Plaza, S.M., 2002. Mitochondrial factors in the pathogenesis of diabetes: a hypothesis for treatment. Altern. Med. Rev. 7, 94-111.

Lass, A., Kwong, L., Sohal, R.S., 1999. Mitochondrial Coenzyme Q content and aging. BioFactors 9, 199-205.

Oliveira, P.J., Santos, D.L., Moreno, A.J.M., 2000. Carvedilol inhibits the exogenous nadh dehydrogenase in rat heart mitochondria. Arch. Biochem. Biophys. 374, 279-285.

Oliveira, P.J., Rolo, A.P., Seiça, R., Palmeira, C.M., Santos, M.S., Moreno, A.J.M., 2001. Decreased susceptibility of heart mitochondria from diabetic GK to mitochondrial transition induced by calcium phosphate. Biosc. Rep. 21, 45-53.

Paulson, D.J., 1997. The diabetic heart is more sensitive to Ischemic injury. Cardiovasc. Res. 34, 104-112.

Perucca-Lostanlen, D., Narbonne, H., Hernandez, J.B., Staccini, P., Saunieres, A., Paquis-Flucklinger, V., et al., 2000. Mitochondrial DNA variations in patients with maternally inherited 
diabetes and deafness syndrome. Biochem. Biophys. Res. Commun. 277, 771-775.

Pierce, G.N., Maddaford, T.G., Russell, J.C., 1997. Cardiovascular dysfunction in insulin-dependent and non-insulin-dependent animal models of diabetes mellitus. Can. J. Physiol. Pharmacol. $75,343-350$.

Schmidt, A.M., Stern, D., 2000. A radical approach to the pathogenesis of diabetic complications. TiPS 21, 367-369.
Takada, M., Ikenoya, S., Yuzuriha, T., Katayama, K., 1984. Simultaneous determination of reduced and oxidized ubiquinones. Methods Enzymol. 105, 147-155.

Takayanagi, R., Takeshige, T., Minakami, P., 1980. NADH- and NADPH-dependent lipid peroxidation in bovine submitochondrial particles. Biochem. J. 192, 853-860.

Zoratti, M., Szabò, I., 1995. The mitochondrial permeability transition. Biochim. Biophy. Acta 1241, 139-176. 